\title{
Suicide rate higher for indigenous people in Labrador
}

A new study on suicide rates in Labrador found a disproportionately higher number of deaths by suicide in Inuit communities than in the general population of Newfoundland and Labrador.

"The results of this study support the need for long-term investments in multilevel suicide-prevention programs targeted at communities with high suicide rates and high-risk groups such as youth," says Dr. Michael Jong, vice-president of medical services with Labrador-Grenfell Health and one of the study's authors.

The study, published in the American Journal of Public Health, found that over a 17-year period, the suicide rate in Newfoundland and Labrador was 8 deaths per 100000 personyears. The rate in Inuit communities was 20 times higher, 165 deaths per 100000 person-years, and the rate for Innu First Nations communities was 14 times higher than the provincial average, at 114 deaths per 100000 person-years.

In addition, $75 \%$ of all suicide deaths in Inuit communities involved men or boys. "However, the suicide rate among Inuit women was still 31 times higher than the rate among women in Newfoundland," notes Jong, who is also president of the Canadian Society for Circumpolar Health.

He points out that the study has important implications for physicians, particularly those working in Northern communities. "When we understand the epidemiology, we can better channel

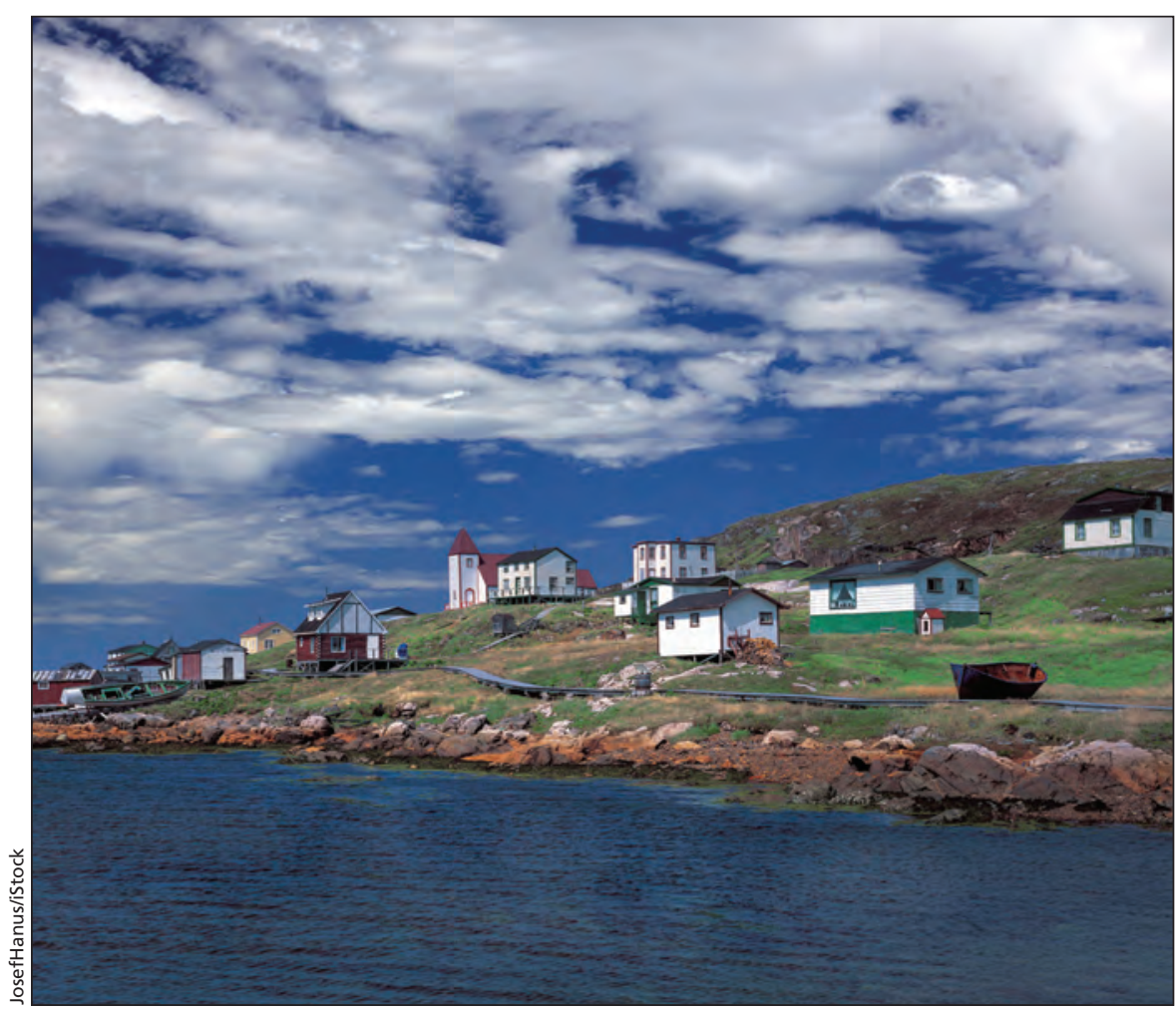

The suicide rate in Inuit communities is $\mathbf{2 0}$ times higher than elsewhere in Newfoundland and Labrador.

our efforts in suicide prevention. Our suicide-prevention [programs] have to address the specific needs of young Inuit and Innu."

Community-led programming that addresses the loss of culture, the teaching of traditional skills and reaffirms a sense of belonging is one of the solutions showing great promise, says Jong. In 2014-15, the Labrador-Grenfell regional health authority completed an extensive environmental scan of suicide- prevention best practices that resulted in a suicide risk-management protocol. "The goal is to have all front-line health care professionals trained in the use of the same processes for assessment, monitoring, treatment and transfer of care for clients who present with suicidal ideation or suicide attempt," says Jong. - donalee Moulton, Halifax, NS

CMAJ 2016. DOI:10.1503/cmaj.109-5305 